R. Delaware, Department of Mathematics and Statistics, University of Missouri-Kansas City, Kansas City, MO 64110. e-mail:

71237.110@compuserve.com

L. Eifler, Department of Mathematics and Statistics, University of

Missouri-Kansas City, Kansas City, MO 64110. e-mail: eiflerl@umkc.edu

\title{
GRAPHS OF FUNCTIONS, REGULAR SETS AND S-STRAIGHT SETS
}

\begin{abstract}
A subset $E$ of $\mathbb{R}^{p}$ is s-straight if $E$ has finite Hausdorff s-dimensional outer measure which equals its Method I s-outer measure. The graph of a continuously differentiable function is shown to be the countable union of closed 1-straight sets together with a set of Hausdorff 1-measure zero. This result is extended to the graphs of absolutely continuous functions and to regular sets.
\end{abstract}

\section{Introduction}

In [6], Foran introduced the notion of s-straight and proposed a subset $E$ of the unit circle such that $E$ is 1-straight and has positive measure. A detailed analysis of this proposed set is given in [2] together with other examples and results for 1-straight sets.

Given a nonempty bounded subset $B$ of $\mathbb{R}^{p}$, define $\operatorname{diam}(B)=\sup \{d(x, y)$ : $x, y \in B\}$ where $d(x, y)$ denotes the usual distance function in $\mathbb{R}^{p}$. Define $\operatorname{diam}(\emptyset)=0$. We write $\operatorname{diam}^{s}(E)$ in place of $[\operatorname{diam}(E)]^{s}$.

Definition 1.1. Let $E$ be a subset of $\mathbb{R}^{p}$ and $s>0$. Given $\infty \geq \delta>0$, define

$$
\bar{m}_{\delta}^{s}(E)=\inf \left\{\sum_{i=1}^{\infty} \operatorname{diam}^{s}\left(E_{i}\right): E=\bigcup_{i=1}^{\infty} E_{i}, \operatorname{diam}\left(E_{i}\right)<\delta \text { for } i=1,2, \ldots\right\} \text {. }
$$

Set $\bar{m}^{s}(E)=\sup _{\delta>0} \bar{m}_{\delta}^{s}(E)$ and set $\bar{m}_{I}^{s}(E)=\bar{m}_{\infty}^{s}(E)$.

Key Words: Hausdorff measure, s-straight sets, regular sets

Mathematical Reviews subject classification: 28A78, 28A05

Received by the editors August 10, 2000 
The outer measure $\bar{m}_{I}^{s}$ is known as a Method I outer measure. The outer measure $\bar{m}^{s}$ is a metric outer measure on $\mathbb{R}^{p}$. Hence, every closed subset of $\mathbb{R}^{p}$ is $\bar{m}^{s}$-measurable. See [3, pp. 132-144] for details. If $E$ is an $\bar{m}^{s}$-measurable subset of $\mathbb{R}^{p}$, then we write $\mathcal{H}^{s}(E)$ in place of $\bar{m}^{s}(E)$. If $\left(A_{n}\right)_{n=1}^{\infty}$ is a decreasing sequence of compact subsets of $\mathbb{R}^{p}$ and if $\delta>0$, then $\bar{m}_{\delta}^{s}\left(\bigcap_{n=1}^{\infty} A_{n}\right)=\lim _{n \rightarrow \infty} \bar{m}_{\delta}^{s}\left(A_{n}\right)$. In [6], Foran defines s-straight and proves Theorem 1.3, providing a useful equivalent formulation of s-straight.

Definition 1.2. Given a subset $E$ of $\mathbb{R}^{p}$ and $s>0$, we say $E$ is s-straight if $\bar{m}^{s}(E)<\infty$ and $\bar{m}^{s}(E)=\bar{m}_{I}^{s}(E)$.

Theorem 1.3. Let $E$ be a subset of $\mathbb{R}^{p}$ with $\bar{m}^{s}(E)<\infty$. Then $E$ is s-straight if and only if $\bar{m}^{s}(E \cap K) \leq \operatorname{diam}^{s}(E \cap K)$ for each compact subset $K$ of $\mathbb{R}^{p}$.

\section{Graphs of Functions and S-Straight Sets}

Definition 2.1. Let $A_{1}, \ldots, A_{n}$ be subsets of $\mathbb{R}^{p}$. We say that $A_{1}, \ldots, A_{n}$ are s-aligned if $\operatorname{diam}^{s}(B) \geq \sum_{i=1}^{n} \operatorname{diam}^{s}\left(B \cap A_{i}\right)$ for each bounded subset $B$ of $A_{1} \cup \cdots \cup A_{n}$.

Example. If $\operatorname{diam}(A \cup B) \geq \operatorname{diam}(A)+\operatorname{diam}(B)$, then $A$ and $B$ may not be 1-aligned. Let $A=\{(x, \sin x): 0 \leq x \leq \pi\}$ and $B=\{(x, \sin x):-\pi \leq x \leq 0\}$. Then $\operatorname{diam}(A \cup B)=\operatorname{diam}(A)+\operatorname{diam}(B)$. If $A_{1}=\{(x, \sin x): 0 \leq x \leq \pi / 2\}$, then $\operatorname{diam}\left(A_{1} \cup B\right)<\operatorname{diam}\left(A_{1}\right)+\operatorname{diam}(B)$. Hence, $A$ and $B$ are not 1-aligned.

The motivation for the definition of s-aligned arises from the following result.

Proposition 2.2. Let $A_{1}, \ldots, A_{n}$ be s-aligned subsets of $\mathbb{R}^{p}$. If $A_{1}, \ldots, A_{n}$ are s-straight, then $A_{1} \cup \cdots \cup A_{n}$ is s-straight.

Proof. Let $K$ be a compact set in $\mathbb{R}^{p}$. Then $\bar{m}^{s}\left[K \cap\left(A_{1} \cup \cdots \cup A_{n}\right)\right] \leq$ $\sum_{i=1}^{n} \bar{m}^{s}\left(K \cap A_{i}\right) \leq \sum_{i=1}^{n} \operatorname{diam}^{s}\left(K \cap A_{i}\right) \leq \operatorname{diam}^{s}\left[K \cap\left(A_{1} \cup \cdots \cup A_{n}\right)\right]$.

Suppose $A$ and $B$ are s-aligned subsets of $\mathbb{R}^{p}$. Let $\bar{A}$ and $\bar{B}$ denote the closure of $A$ and $B$, respectively. Then $\bar{A} \cap \bar{B}$ contains at most one point. Hence, $\bar{m}^{s}\left(A_{1} \cup B_{1}\right)=\bar{m}^{s}\left(A_{1}\right)+\bar{m}^{s}\left(B_{1}\right)$ for each $A_{1} \subseteq \bar{A}$ and $B_{1} \subseteq \bar{B}$.

Theorem 2.3. Let $\left(q_{n}\right)_{n=1}^{\infty}$ be a sequence of positive integers. Set $Q^{n}=$ $\left\{\left(i_{1}, \ldots, i_{n}\right): 1 \leq i_{1} \leq q_{1}, \ldots, 1 \leq i_{n} \leq q_{n}\right\}$ for $n=1,2, \ldots$ Let $\left\{A_{\beta}: \beta \in\right.$ $Q^{n}$ for some $\left.n \geq 1\right\}$ be a family of compact subsets of $\mathbb{R}^{p}$ such that $A_{1}, \ldots, A_{q_{1}}$ are s-aligned and such that

(a) $A_{(\beta, 1)}, \ldots, A_{\left(\beta, q_{n}\right)}$ are s-aligned if $\beta \in Q^{n-1}$,

(b) $A_{\beta} \supseteq A_{(\beta, 1)} \cup \cdots \cup A_{\left(\beta, q_{n}\right)}$ if $\beta \in Q^{n-1}$ and 
(c) $\lim _{n \rightarrow \infty} \max \left\{\operatorname{diam}^{s}\left(A_{\beta}\right): \beta \in Q^{n}\right\}=0$.

Let $P_{n}=\bigcup\left\{A_{\beta}: \beta \in Q^{n}\right\}$. Then $\bigcap_{n=1}^{\infty} P_{n}$ is s-straight.

Proof. If $E \subseteq P_{n}$, then $\operatorname{diam}^{s}(E) \geq \sum_{\beta \in Q^{n}} \operatorname{diam}^{s}\left(E \cap A_{\beta}\right)$. Let $P=$ $\bigcap_{n=1}^{\infty} P_{n}$. The set $P$ is closed and $P_{n} \supseteq P_{n+1}$ for $n \geq 1$. Let $K$ be a compact subset of $\mathbb{R}^{p}$. It suffices to show that $\operatorname{diam}^{s}(P \cap K) \geq \bar{m}_{\delta}^{s}(P \cap K)$ for each $\delta>0$. Let $\delta>0$. Choose $\ell$ such that $\operatorname{diam}^{s}\left(A_{\beta}\right)<\delta$ for each $\beta \in Q^{\ell}$. Then for each $n \geq \ell$

$$
\operatorname{diam}^{s}\left(P_{n} \cap K\right) \geq \sum_{\beta \in Q^{n}} \operatorname{diam}^{s}\left(A_{\beta} \cap K\right) \geq \bar{m}_{\delta}^{s}\left(P_{n} \cap K\right) .
$$

Hence, $\lim _{n \rightarrow \infty} \operatorname{diam}^{s}\left(P_{n} \cap K\right) \geq \lim _{n \rightarrow \infty} \bar{m}_{\delta}^{s}\left(P_{n} \cap K\right) \geq \bar{m}_{\delta}^{s}(P \cap K)$. Since $P$ is closed, $\operatorname{diam}^{s}(P \cap K)=\lim _{n \rightarrow \infty} \operatorname{diam}^{s}\left(P_{n} \cap K\right)$. Thus, $\operatorname{diam}^{s}(P \cap K) \geq$ $\bar{m}_{\delta}^{s}(P \cap K)$. It follows that $P$ is s-straight.

Lemma 2.4. Let $f:[a, b] \rightarrow \mathbb{R}$ be continuously differentiable with $0 \leq m \leq$ $f^{\prime}(t) \leq M$. If $I$ is a subinterval of $[a, b]$, let $F(I)=\{(x, f(x)): x \in I\}$. Suppose $0<w<1$ satisfies $(1+w) \sqrt{1+m^{2}} \geq 2 w \sqrt{1+M^{2}}$. Then for each positive integer $n$, there exist disjoint subintervals $I_{1}, \ldots, I_{n}$ of $[a, b]$ each of length $w(b-a) / n$ such that $\mathcal{H}^{1}\left[\bigcup_{k=1}^{n} F\left(I_{k}\right)\right] \geq w^{2} \mathcal{H}^{1}[F([a, b])]$ and $F\left(I_{1}\right), \ldots, F\left(I_{n}\right)$ are 1-aligned.

Proof. It suffices to consider the case where $[a, b]=[0,1]$. Let $F(s, t)=$ $\{(x, f(x)): s \leq x \leq t\}$ if $0 \leq s \leq t \leq 1$. Assume $(1+w) \sqrt{1+m^{2}} \geq$ $2 w \sqrt{1+M^{2}}$ where $0<w<1$. Set $\alpha=\arctan m$ and $\beta=\arctan M$. Then $\tan \alpha \leq f^{\prime} \leq \tan \beta$ and $(1+w) \sec \alpha \geq 2 w \sec \beta$. If $0 \leq s \leq t \leq 1$, then $(t-s) \sec \alpha \leq \mathcal{H}^{1}[F(s, t)] \leq(t-s) \sec \beta$. Let $n$ be a positive integer. Set $\delta=(1-w) /(2 n)$. Define $a_{k}=(k-1) / n+\delta, b_{k}=k / n-\delta$ and $I_{k}=\left[a_{k}, b_{k}\right]$ for $k=1, \ldots, n$. Then $\mathcal{H}^{1}\left[\bigcup_{k=1}^{n} F\left(I_{k}\right)\right]=\sum_{k=1}^{n} \mathcal{H}^{1}\left[F\left(I_{k}\right)\right] \geq \sum_{k=1}^{n}\left|I_{k}\right| \sec \alpha=$ $w \sec \alpha$ where $\left|I_{k}\right|$ denotes the length of $I_{k}$. Hence

$$
\mathcal{H}^{1}\left[\bigcup_{k=1}^{n} F\left(I_{k}\right)\right] \geq w \frac{\sec \alpha}{\sec \beta} \mathcal{H}^{1}[F(0,1)] \geq \frac{2 w^{2}}{1+w} \mathcal{H}^{1}[F(0,1)] \geq w^{2} \mathcal{H}^{1}[F(0,1)] .
$$

Since $f$ is increasing, to show that $F\left(I_{1}\right), \ldots, F\left(I_{n}\right)$ are 1-aligned, it suffices to show that $\operatorname{diam}[F(s, t)] \geq \sum_{k=1}^{n} \operatorname{diam}\left[F\left([s, t] \cap I_{k}\right)\right]$ if $0 \leq s<t \leq 1$. If $s, t$ belong to a single $I_{k}$, this inequality is clear. Let $a_{j} \leq s<b_{j}$ and let $a_{k}<t \leq b_{k}$ where $j<k$. Set

$\Phi(s, t)=\operatorname{diam}[F(s, t)]-\operatorname{diam}\left[F\left(s, b_{j}\right)\right]-\operatorname{diam}\left[F\left(a_{k}, t\right)\right]-\sum_{i=j+1}^{k-1} \operatorname{diam}\left[F\left(a_{i}, b_{i}\right)\right]$. 
To show that $F\left(I_{1}\right), \ldots, F\left(I_{n}\right)$ are 1-aligned, it suffices to show that $\Phi(s, t) \geq$ 0 . Set $m=k+1-j \geq 2$. We have

$$
\begin{aligned}
\Phi(s, t) & \geq(t-s) \sec \alpha-\left[\left(b_{j}-s\right)+\left(t-a_{k}\right)+\sum_{i=j+1}^{k-1}\left(b_{i}-a_{i}\right)\right] \sec \beta \\
& \geq\left(b_{k}-a_{j}\right) \sec \alpha-\left[\left(b_{j}-a_{j}\right)+\left(b_{k}-a_{k}\right)+\sum_{i=j+1}^{k-1}\left(b_{i}-a_{i}\right)\right] \sec \beta \\
& =\frac{m-(1-w)}{n} \sec \alpha-\frac{m w}{n} \sec \beta \\
& =\frac{m}{n}\left[\frac{m-1+w}{m} \sec \alpha-w \sec \beta\right] \\
& \geq \frac{m}{n}\left[\frac{1+w}{2} \sec \alpha-w \sec \beta\right] .
\end{aligned}
$$

Hence, $\Phi(s, t) \geq 0$ and so $F\left(I_{1}\right), \ldots, F\left(I_{n}\right)$ are 1-aligned.

Theorem 2.5. Let $f:[a, b] \rightarrow \mathbb{R}$ be continuously differentiable with $0 \leq f^{\prime} \leq$ M. Let $\Gamma=\{(x, f(x)): a \leq x \leq b\}$. Then there exists closed 1-straight subset $P$ of $\Gamma$ such that $\mathcal{H}^{1}(P) \geq \mathcal{H}^{1}(\Gamma) /\left[4\left(1+M^{2}\right)\right]$.

Proof. If $I$ is a subinterval of $[a, b]$, set $F(I)=\{(x, f(x)): x \in I\}, m\left(f^{\prime}, I\right)=$ $\min \left\{f^{\prime}(x): x \in I\right\}$ and $M\left(f^{\prime}, I\right)=\max \left\{f^{\prime}(x): x \in I\right\}$. Set $\beta=\arctan (M)$ and let $w_{1}$ be the positive number such that $1+w_{1}=2 w_{1} \sec \beta$, that is, $w_{1}=1 /(2 \sec \beta-1)$. Then $w_{1}^{2}>1 /\left[4\left(1+M^{2}\right)\right]$. Choose $w_{2}, w_{3}, \ldots$ in $(0,1)$ such that $\prod_{k=1}^{\infty} w_{k}^{2}=1 /\left[4\left(1+M^{2}\right)\right]$. By Lemma 2.4 and by uniform continuity of $f^{\prime}$ on $[a, b]$, we may choose an integer $q_{1} \geq 2$ and closed subintervals $I_{1}, \ldots, I_{q_{1}}$ of $[a, b]$ each of length $w_{1}(b-a) / q_{1}$ such that

(step 1) $\left\{\begin{array}{l}\mathcal{H}^{1}\left[\bigcup_{j=1}^{q_{1}} F\left(I_{j}\right)\right] \geq w_{1}^{2} \mathcal{H}^{1}(\Gamma) \text { and } \\ \frac{1+w_{2}}{2 w_{2}} \sqrt{\frac{1+m^{2}\left(f^{\prime}, I_{j}\right)}{1+M^{2}\left(f^{\prime}, I_{j}\right)}} \geq 1 \text { for } j=1, \ldots, q_{1}\end{array}\right.$

Let $P_{1}=F\left(I_{1}\right) \cup \cdots \cup F\left(I_{q_{1}}\right)$. Then $\mathcal{H}^{1}\left(P_{1}\right) \geq w_{1}^{2} \mathcal{H}^{1}(\Gamma)$. By Lemma 2.4 and by uniform continuity of $f^{\prime}$ on $[a, b]$, we may choose an integer $q_{2} \geq 2$ and closed subintervals $I_{i, 1}, \ldots, I_{i, q_{2}}$ of $I_{i}$ each of length $w_{1} w_{2}(b-a) /\left(q_{1} q_{2}\right)$ for $i=1, \ldots, q_{1}$ such that

(step 2) $\left\{\begin{array}{l}F\left(I_{i, 1}\right), \ldots, F\left(I_{i, q_{2}}\right) \text { are 1-aligned for } i=1, \ldots, q_{1} \\ \mathcal{H}^{1}\left[\bigcup_{j=1}^{q_{2}} F\left(I_{i, j}\right) \geq w_{2}^{2} \mathcal{H}^{1}\left[F\left(I_{i}\right)\right] \text { for } i=1, \ldots, q_{1} \text { and }\right. \\ \frac{1+w_{3}}{2 w_{3}} \sqrt{\frac{1+m^{2}\left(f^{\prime}, I_{i, j}\right)}{1+M^{2}\left(f^{\prime}, I_{i, j}\right)}} \geq 1 \text { for } i=1, \ldots, q_{1} \text { and } j=1, \ldots, q_{2}\end{array}\right.$ 
Let $P_{2}=\bigcup\left\{F\left(I_{i, j}\right): 1 \leq i \leq q_{1}\right.$ and $\left.1 \leq j \leq q_{2}\right\}$. Then $\mathcal{H}^{1}\left(P_{2}\right) \geq$ $w_{1}^{2} w_{2}^{2} \mathcal{H}^{1}(\Gamma)$. Continuing this process, we obtain, in accordance with Theorem 2.3, a decreasing sequence $\left(P_{n}\right)_{n=1}^{\infty}$ of compact subsets of $\Gamma$ such that $\mathcal{H}^{1}\left(P_{n}\right) \geq\left(\prod_{k=1}^{n} w_{k}^{2}\right) \mathcal{H}^{1}(\Gamma)$ for each $n \geq 1$ and $\bigcap_{n=1}^{\infty} P_{n}$ is 1-straight. Let $P=\bigcap_{n=1}^{\infty} P_{n}$. Then $P$ is a closed 1-straight subset of $\Gamma$ with $\mathcal{H}^{1}(P) \geq$ $\mathcal{H}^{1}(\Gamma) /\left[4\left(1+M^{2}\right)\right]$.

Let $f:[a, b] \rightarrow \mathbb{R}$ be continuous and let $\Gamma$ be the graph of $f$. Assume that $\mathcal{H}^{1}(\Gamma)$ is finite. If each closed subset of $\Gamma$ with positive $\mathcal{H}^{1}$ measure contains a closed 1-straight subset of positive $\mathcal{H}^{1}$ measure, then $\Gamma$ is the countable union of closed 1-straight subsets together with a set of $\mathcal{H}^{1}$ measure zero since the measure $\mathcal{H}^{1}$ is regular. See [2] for a similar result.

Corollary 2.6. Let $f:[a, b] \rightarrow \mathbb{R}$ be continuously differentiable. Then the graph of $f$ is the countable union of closed 1-straight sets together with a set of $\mathcal{H}^{1}$ measure zero.

Proof. If $I$ is a subinterval of $[a, b]$, let $F(I)=\{(x, f(x)): x \in I\}$. Choose closed subintervals $I_{1}, \ldots, I_{n}$ of $[a, b]$ that cover $[a, b]$ such that $f^{\prime} \geq 0, f^{\prime} \leq 0$ or $\left|f^{\prime}\right| \leq \sqrt{3} / 3$ on $I_{k}$ for each $k=1, \ldots, n$. If $\left|f^{\prime}\right| \leq \sqrt{3} / 3$ on $I_{j}$, then $F\left(I_{j}\right)$ rotated by $30^{\circ}$ coincides with the graph of a continuously differentiable function $g$ with $g^{\prime} \geq 0$. It follows from Theorem 2.5 that each $F\left(I_{k}\right)$ is the countable union of closed 1-straight subsets together with a subset of $\mathcal{H}^{1}$ measure zero and so likewise for the graph of $f$.

Theorem 2.7. Let $f:[a, b] \rightarrow \mathbb{R}$ be absolutely continuous. Then the graph of $f$ is the countable union of closed 1-straight sets together with a set of $\mathcal{H}^{1}$ measure zero.

Proof. Let $\Gamma_{f}=\{(x, f(x)): a \leq x \leq b\}$. Let $\mu$ denote Lebesgue measure on $[a, b]$. Let $E$ be a closed subset of $\Gamma_{f}$ with positive $\mathcal{H}^{1}$ measure. Set $B=\{x \in[a, b]:(x, f(x)) \in E\}$. Since $f$ is absolutely continuous, $B$ is a compact subset of $[a, b]$ with $\mu(B)>0$. But $f$ is differentiable almost everywhere and $f^{\prime}$ is $\mu$-measurable. By a theorem due to Federer, see [5, Theorem 3.1.15] and [1, p. 1160], there exists a compact subset $K$ of $B$ and a continuously differentiable function $g$ on $[a, b]$ such that $g=f$ on $K$ and $\mu(K)>0$. Let $\Gamma_{g}(K)=\{(x, g(x)): x \in K\}$. Then $\Gamma_{g}(K)$ is a compact subset of the graph of $g$ with positive $\mathcal{H}^{1}$ measure. By Corollary 2.6, there exists a closed 1-straight subset $P$ of the graph of $g$ with $\mathcal{H}^{1}\left(P \cap \Gamma_{g}(K)\right)>0$. Since $P$ is 1-straight, $P \cap \Gamma_{g}(K)$ is a closed 1-straight subset of $E$. Hence, the graph of $f$ is the countable union of closed 1-straight subsets together with a set of $\mathcal{H}^{1}$ measure zero. 
Corollary 2.8. Let $f:[a, b] \rightarrow \mathbb{R}$ be continuous and increasing. Then the graph of $f$ is the countable union of closed 1-straight sets together with a set of $\mathcal{H}^{1}$ measure zero.

Proof. The graph of $f$ rotated clockwise by $45^{\circ}$ coincides with the graph of a continuous Lipschitz function, which is absolutely continuous.

\section{Regular Sets and S-Straight Sets}

If $E \subset \mathbb{R}^{p}$, we say $E$ is an s-set if $E$ is $\bar{m}^{s}$-measurable and $0<\mathcal{H}^{s}(E)<\infty$. If $x \in \mathbb{R}^{p}$, let $B_{r}(x)$ denote the closed ball of radius $r>0$ centered at $x$. We next recall some definitions concerning density, regular sets and contingents.

Definition 3.1. [4, pp. 20-21]. We say $x$ is a regular point of $E$ if the $\mathcal{H}^{s}$-density of $x$ with respect to $E$, defined as $\lim _{r \rightarrow 0^{+}}\left[\mathcal{H}^{s}\left(E \cap B_{r}(x)\right) /(2 r)^{s}\right]$, exists and equals 1. A set $E$ is called a regular set if almost every point of $E$ is regular. Here we consider only the case $s=1$ and sets $E \subseteq \mathbb{R}^{2}$.

Definition 3.2. [4, pp. 26-29]. A curve (or, Jordan curve) $\Gamma$ is the image of a continuous one-to-one function $\psi:[a, b] \rightarrow \mathbb{R}^{p}$, where $[a, b] \subseteq \mathbb{R}$ is a closed interval. In particular, a curve is not self-intersecting. If the length $\mathcal{L}(\Gamma)$ of a curve $\Gamma$ is defined is the usual way and if $\mathcal{L}(\Gamma)<\infty$, we say that $\Gamma$ is a rectifiable curve. A 1-set contained in a countable union of rectifiable curves is called a $Y$-set [4, p. 33]. Let $S(x, \theta, \varphi)$ be the closed one-way infinite cone with vertex $x$ and axis in direction of angle $\theta$ consisting of those points $y$ such that the line segment between $x$ and $y$ makes an angle of at most $\varphi$ with that axis.

Definition 3.3. [7, p. 262]. Let $E \subseteq \mathbb{R}^{2}$. For any point $x \in E$, the direction $\theta$ of a half-line originating at $x$ is the angle made by that half-line with a fixed direction, usually that of the horizontal axis. Such a half-line is denoted by $l(x, \theta)$. A half-line containing a point $y \neq x$ is denoted by $\overrightarrow{x y} . A$ sequence $\left\{l_{n}\left(x, \theta_{n}\right)\right\}_{n=1}^{\infty}$ of half-lines is said to converge to the half-line $l(x, \theta)$ if $\lim _{n \rightarrow \infty} \theta_{n}=\theta$. A half-line $l(x, \theta)$ is called an intermediate half-tangent of $E$ at $x$ if there exists a sequence of points $\left\{x_{n}\right\}_{n=1}^{\infty} \subseteq E$, with $x_{n} \neq x$ for all $n$ such that both $\lim _{n \rightarrow \infty} x_{n}=x$, and the sequence of half-lines $\left\{\overrightarrow{x x_{n}}\right\}_{n=1}^{\infty}$ converges to $l(x, \theta)$. Finally, the contingent of $E$ at $x$, denoted by $\operatorname{contg}_{E}(x)$, is the set of all intermediate half-tangents of $E$ at $x$. (If $x_{0}$ is an isolated point, then $x_{0}$ has no intermediate half-tangents, and $\operatorname{contg}_{E}\left(x_{0}\right)=\emptyset$.)

The following Lemma is part of the so-called Fundamental Theorem on Contingents of Plane Sets, found in Saks. 
Lemma 3.4. [7, p. 264]. Let $\theta$ be a fixed direction, and $E \subseteq \mathbb{R}^{2}$ be such that for each $x \in E$, the set $\operatorname{contg}_{E}(x)$ contains no half-line of direction $\theta$. If $\theta$ is the direction of the positive vertical axis, then $E=\cup_{i=1}^{\infty} E_{i}$ such that for each $i$ the set $E_{i}$ is the graph of a Lipschitz function $f_{i}: B_{i} \rightarrow \mathbb{R}$ where $B_{i}$ is a bounded subset of $\mathbb{R}$ and $\mathcal{H}^{1}\left(E_{i}\right)=\mathcal{L}\left(E_{i}\right)<\infty$.

Theorem 3.5. Let $E \subseteq \mathbb{R}^{2}$ be a regular 1-set. Then $E$ is a countable union of 1-straight sets together with a set of $\mathcal{H}^{1}$ measure zero.

Proof. Besicovitch proved (see [4, p. 45]) that a regular 1-set $E \subseteq \mathbb{R}^{2}$ consists of a $Y$-set together with a set of $\mathcal{H}^{1}$-measure zero. In his proof (see [4, p. 32]) that a rectifiable curve $\Gamma$ in $\mathbb{R}^{2}$ has a tangent at almost all of its points, he proved in particular that for almost all $x \in \Gamma$ there exists a direction $\theta$ such that for suitable $\varphi, \rho>0$ it follows that $\Gamma \cap\left[B_{\rho}(x) \backslash(S(x, \theta, \varphi) \cup S(x, \theta+\right.$ $\pi, \varphi))]=\emptyset$. So at almost all points $x$ of a rectifiable curve $\Gamma, \operatorname{contg}_{\Gamma}(x) \neq \mathbb{R}^{2}$. As in the proof of the Fundamental Theorem on Contingents of Plane Sets [7, p. 267], let $\left\{\theta_{n}\right\}$ be a countable everywhere dense set of directions in $\mathbb{R}^{2}$. Let $\Gamma_{n}$ be the set of points of $\Gamma$ at which $\operatorname{contg}_{\Gamma}(x)$ does not contain the half-line of direction $\theta_{n}$. Then since $\left\{\theta_{n}\right\}$ is dense, it is clear that $\Gamma=$ $\cup_{n=1}^{\infty} \Gamma_{n}$, otherwise there exist points $x \in \Gamma$ such that $\operatorname{contg}_{\Gamma}(x)=\mathbb{R}^{2}$. By Lemma 3.4, with respect to a line of direction $\theta_{n}+\frac{\pi}{2}$, each $\Gamma_{n}$ is the countable union of finite length graphs of Lipschitz functions on bounded domain sets. Thus $\Gamma$ itself equals such a union. It is well-known, as in [7, p. 264], that any such Lipschitz function can be extended to be Lipschitz on the smallest closed interval containing its bounded domain. Since a Lipschitz function is absolutely continuous, by Theorem 2.7 it follows that the graph of each such extended Lipschitz function is a countable union of 1-straight sets. Since subsets of 1-straight sets are 1-straight and since translations and rotations of 1-straight sets are 1-straight (see [2]), the subset $\Gamma$ of the countable union of the graphs of these Lipschitz functions is also a countable union of 1-straight sets. Since this is true for each rectifiable curve $\Gamma$ in $\mathbb{R}^{2}$, it follows that $E$ is therefore a countable union of 1 -straight sets together with a set of $\mathcal{H}^{1}$-measure zero.

Acknowledgment. The authors kindly thank James Foran for valuable conversations about s-straight sets.

\section{References}

[1] J. B. Brown, Intersections of continuous, Lipschitz, Hölder class and smooth functions, Proc. Amer. Math. Soc. 123(4), pp. 1157-1165. 
[2] R. Delaware, Sets whose Hausdorff measure equals Method I outer measure, Ph.D. Dissertation, University of Missouri-Kansas City, 2000.

[3] G. A. Edgar, Measure, topology, and fractal geometry, Springer-Verlag, 1990.

[4] K. J. Falconer, The geometry of fractal sets, Cambridge University Press, 1985.

[5] H. Federer, Geometric measure theory, Springer-Verlag, 1969.

[6] J. Foran, Measure preserving continuous straightening of fractional dimensional sets, Real Analysis Exchange 21(2), 1995/6, pp. 732-738.

[7] S. Saks, Theory of the integral, 2nd Revised Edition, Dover, 1964. 